Schweiz. Z. Path. Bakt. 1942;5:I-IV

\title{
Contents, Vol. 5, 1942
}

Schweizerische Zeitschrift

für

Pathologie und Bakteriologie

Revue Suisse de Pathologie et de Bactériologie

Unter Mitarbeit von: C. Hallauer P. Hauduroy H. v. Meyenburg J. L. Nicod Th. Reh

Bern Lausanne Zurich Lausanne Geneve

E. Rutishauser C. Wegelin A. Werthemann

Geneve Bern Basel

Herausgegeben von:

A. v. Albertini - A. Grumbach - H. Mooser

Zurich

Vol. V

1942

BASEL (Schweiz) S. K A R G E R NEW YORK

Holbei $\pi$ strasse $22 \quad 215$ Fourth Avenue

$\mathrm{vO}^{1 / 8}$

$\mathrm{S}$.

$\frac{89^{\circ}}{10}$

Alle Rechte vorbehalten

Printed in Switzerland.

Clidiés: Aberegg-Steiner \& Cie. AC, Bern.

Uruck von Friedrich Reinhardt in Basel.

Index

Ackermann, H., Beitrag zur Kenntnis der cavernösen Haemangiome des Ovariums

79

Albertini, A. v., Weitere Beiträge zur Pathogenese der idiopathischen Pachymeningitis haemorrhagica interna. 293

Aygün, S. T. und Aygün, K. S., Experimentelle und pathologisch-anatomische -Studien über Pleuro-Pneumonia contagiosa Caprae in Anatolien und ihre Beziehungen zur menschlichen Grippe 216

Beumer, J'., vide Bordet, P.

Bloch, H., Die Beziehungen der Bakteriophagen zu den

Virusarten Supplementum 
Bordet, P., et Beumer, J., Correlation entre la reproduction du bactériophage et la formation de substance inhibitrice par la bactérie sensible 265

Bream, H. und E. K. Unat, Ueber thermolabile Leibesantigene des Ruhrbazillus Flexner

Bueding, E., vide Ladewig, P.

Kallós, P., Zur Frage der Organspezifität von Nierenex-

trakten 119

Kallós, P., und Kallós-Deffner, L., Beiträge zum Verständnis des allergischen Bronchialasthmas .... 97

Ladewig, P. und Bueding, E., Funktionell-morphologische Studie an Meerschweinchenlebern bei subakuter Phos phor- und Chlorol'ornivergiftung 178

Maskar, Ue., Ueber Karzinonie der Analgegend, besonders der Zirkumanaldrüsen, beim Hunde .... 329

Meier, A., Gibt es einen Zuckerkrebs? 226

Miescher, G., Züchtung von Gonokokken auf Vitamin-B!haltigen Nährböden 388

Muehlon, W. E., Zur Kenntnis des Malherbe'schen Epithelioms 53

Reimann, F., Ueber die Beziehung der Retikulozyten zur Hämoglobinbildung und zum erhöhten Gehalt an Blutfarbstoff im Blut der perniziösen Anämie . . . 343

Schnetz, A., Züchtung von Gonokokken auf Vitamin-B-haltigen Nährböden 238

Senarclens, F. de, Granulomatose lipoïdique hépatique expérimentale chez le rat 150

Skorpil, F., Eine ungewöhnliche Form von Hautretikulose 310

Spitzer, R., Untersuchungen über die Gewöhnung von Gonokokken an Sulfanilamide (Cibazol und Sulfanilamid) und andere Bakteriengifte (Trypaflavin und Oxycyanat) im Kulturexperiment 275 Turchini, J., Un nouveau moyen d'investigation physique applique à $\Gamma$ histologie. La radiographie histologique .137 Unat, E. K., vide Brawn, H.

Viollier, G., Die Histologie der experimentellen Fleckfieberpneumonie weißer Mäuse 360

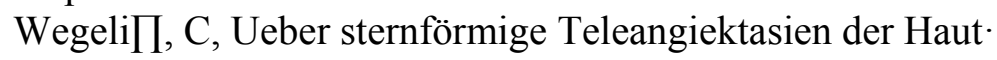
bei Lebercirrhose $\quad 374$

Wohlwill, F., Untersuchungen über die Gewebsreaktion auf Thorotrast bei Anwendung dieser Substanz zu diagnostischen Zwecken am Menschen 21 UEBERSICHTSREFERATE - REVIEWS - REVUES

Hallauer, C, Ergebnisse der Endotoxinforschung . 122 Hauduroy, P., et Posternak, J., Les applications bactériologiques de la microscopie en fluorescence. Recherches 
sur la microscopie en fluorescence du bacille tubercu-

leux

240

Posternak, J., vide Hauduroy, P.

BUCHBESPRECHUNGEN - BOOKS REVIEW -LIVRES NOUVEAUX

135,263 